\title{
Distributed Coordination Algorithms for Multiple Fractional-Order Systems
}

\author{
Yongcan $\mathrm{Cao}^{\dagger}$, Yan $\mathrm{Li}^{\ddagger \dagger}$, Wei Ren ${ }^{\dagger *}$, YangQuan $\mathrm{Chen}^{\dagger}$
}

\begin{abstract}
This paper studies distributed coordination algorithms for multiple fractional-order systems over a directed communication graph. A general fractional-order consensus model is introduced by summarizing three different cases: (i) fractional-order agent dynamics with integer-order consensus algorithms, (ii) fractional-order agent dynamics with fractional-order consensus algorithms, and (iii) integer-order agent dynamics with fractional-order consensus algorithms. We show sufficient conditions on the communication graph and the fractional order such that consensus can be achieved using the general model. The consensus equilibrium is also given explicitly. In addition, we characterize the relationship between the number of agents and the fractional order to ensure consensus. Furthermore, we compare the convergence speed of consensus for fractional-order systems with that for integer-order systems. It is shown that the convergence speed of the fractional-order consensus algorithms can be improved by varying the fractional orders with time. Finally, simulation results are presented as a proof of concept.
\end{abstract}

\section{INTRODUCTION}

Consensus has an old history [1], [2], [3]. In the literature, consensus means agreement of a group faced with decisionmaking situations. As for a group behavior, sharing information with each other, or consulting more than one expert as stated in [1] makes the decision makers more confident.

Inspired by the ideas in the literature, similar strategies have been applied in multi-agent systems recently. For systems with single-integrator dynamics, consensus algorithms have been studied extensively (see [4] and references therein). Because many existing vehicles are modeled by double-integrator dynamics, consensus algorithms for double-integrator dynamics are studied in [5], [6], [7], [8], [9], to name a few.

In reality, consensus phenomena exist widely in biology, economics, social science, etc. However, the study of consensus is restricted to systems with integer-order dynamics at present. In fact, there are many phenomena that cannot or are hard to be interpreted by integer-order dynamics, for example, the consensus motion of agents in viscoelastic materials such as macromolecule fluid, porous media, and complicated environments. In these situations, the stress-strain relationships always show dynamics with some non-integer (fractional) order as shown in [10], [11],

\footnotetext{
†Electrical and Computer Engineering Department, Utah State University, Logan, UT 84322, USA.

$\ddagger$ Institute of Applied Math, School of Mathematics and System Sciences, Shandong University, Jinan 250100, P. R. China. Yan Li was supported in part by the State Scholarship Fund of the P. R. China (Grant No. LiuJinChu[2007]3020-2007102037).

*Corresponding author: wren@engineering.usu.edu. Wei Ren was supported in part by National Science Foundation Grant CNS-0834691.
}

[12]. Fractional-order dynamics can describe many complex phenomena that are beyond the ability of classical integer-order calculus. In particular, there are many other phenomena that can be naturally interpreted by consensus for systems with fractional-order dynamics, for example, chemotaxi behavior and food seeking of microbe, especially the motion of bacteria in lubrications which are perspired by themselves [13], [14].

The contribution of this paper is as follows: First, consensus for integer-order dynamics is extended to fractional-order dynamics. Existing consensus algorithms for integer-order dynamics can be considered a special case of fractionalorder consensus algorithms. Second, we study the conditions on the communication graph and the fractional order such that consensus can be achieved for fractional-order systems. We also characterize the relationship between the number of agents and the fractional order to ensure consensus. Third, an alternative to improve the convergence speed is presented by applying a varying-order consensus strategy.

\section{PRELIMinary}

\section{A. Graph Theory}

For a system with $n$ agents, the communication graph for all agents can be modeled by a directed graph $\mathcal{G}=$ $(\mathcal{V}, \mathcal{W})$, where $\mathcal{V}=\left\{v_{1}, v_{2}, \cdots, v_{n}\right\}$ and $\mathcal{W} \subseteq \mathcal{V}^{2}$ represent, respectively, the agent set and the edge set of the graph. Each edge can be denoted as $\left(v_{i}, v_{j}\right)$ which means agent $j$ can access the state information of agent $i$. Accordingly, agent $i$ is a neighbor of agent $j$. All neighbors of agent $i$ is denoted by $N_{i}$. A directed path is a sequence of edges in a directed graph with the form $\left(v_{1}, v_{2}\right),\left(v_{2}, v_{3}\right), \cdots$, where $v_{i} \in \mathcal{V}$. A directed graph has a directed spanning tree if there exists at least one agent that has a directed path to all other agents.

The communication graph can be represented by two types of matrices: the adjacency matrix $A=\left[a_{i j}\right] \in \mathbb{R}^{n \times n}$ with $a_{i j}>0$ if $\left(v_{j}, v_{i}\right) \in \mathcal{W}$ and $a_{i j}=0$ otherwise, and the (nonsymmetric) Laplacian matrix $L=\left[\ell_{i j}\right] \in \mathbb{R}^{n \times n}$ with $\ell_{i i}=\sum_{j \in N_{i}} a_{i j}$ and $\ell_{i j}=-a_{i j}, i \neq j$. It is straightforward to verify that $L$ has at least one zero eigenvalue with a corresponding eigenvector $\mathbf{1}$, where $\mathbf{1}$ is an all-one column vector with a compatible size.

Lemma 2.1: [15] For a fixed communication graph, $L$ has a simple zero eigenvalue with an associated eigenvector 1 and all other eigenvalues have positive real parts if and only if the communication graph has a directed spanning tree. 


\section{B. Consensus Algorithm for Integer-order Systems}

For an $n$-agent system with single-integrator dynamics

$$
\dot{x}_{i}(t)=u_{i}(t),
$$

where $x_{i}(t) \in \mathbb{R}$ is the state of $i$ th agent and $u_{i}(t) \in \mathbb{R}$ represents the corresponding control input, a fundamental consensus algorithm is given by [16], [17], [15]

$$
u_{i}(t)=\sum_{j \in N_{i}} a_{i j}\left[x_{j}(t)-x_{i}(t)\right]
$$

where $a_{i j}$ is the $(i, j)$ th entry of the adjacency matrix $A$. Using (2), (1) can be written in matrix form as

$$
\dot{X}(t)=-L X(t)
$$

where $X(t)=\left[x_{1}(t), x_{2}(t), \ldots, x_{n}(t)\right]^{T} \in \mathbb{R}^{n}$ and $L$ is the (nonsymmetric) Laplacian matrix.

For high integer-order multi-agent systems, the consensus algorithms are similar to those for the single-integrator systems (see [18] and references therein).

\section{Fractional Calculus}

The Caputo fractional-order derivative plays a crucial role in fractional-order calculus. Caputo integral is defined from Heaviside unit step function as

$$
{ }_{a}^{C} D_{t}^{-\alpha} f(t)=\frac{1}{\Gamma(\alpha)} \int_{a}^{t} \frac{f(\tau)}{(t-\tau)^{1-\alpha}} \mathrm{d} \tau,
$$

where $\Gamma(\cdot)$ is the Gamma function, $\alpha \in(0,1], a$ is an arbitrary real number, and ${ }_{a}^{C} D_{t}^{-\alpha}$ denotes the Caputo integral with order $\alpha$. For an arbitrary real number $p$, Caputo derivative is defined as

$$
{ }_{a}^{C} D_{t}^{p} f(t)={ }_{a}^{C} D_{t}^{-\alpha}\left[\frac{\partial^{[p]+1}}{\partial t[p]+1} f(t)\right],
$$

where $\alpha=[p]+1-p \in(0,1]$ and $[p]$ is the integer part of $p$. If $p$ is an integer, then $\alpha=1$ and (4) is equivalent to the integer-order derivative. Based on the formal definition of the Laplace transform

$$
F(s)=\mathcal{L}\{f(t)\}=\int_{0^{-}}^{\infty} e^{-s t} f(t) d t
$$

where $\mathcal{L}\{\cdot\}$ denotes the Laplace transform of a function, it follows that the Laplace transform $\mathcal{L}\left\{{ }_{a}^{C} D_{t}^{\alpha} f(t)\right\}$ is

$$
\begin{cases}s^{\alpha} F(s)+s^{\alpha-1} f\left(0^{-}\right), & \alpha \in(0,1] \\ s^{\alpha} F(s)+s^{\alpha-1} f\left(0^{-}\right)+s^{\alpha-2} \dot{f}\left(0^{-}\right), & \alpha \in(1,2),\end{cases}
$$

where $f\left(0^{-}\right)=\lim _{\epsilon \rightarrow 0^{-}} f(\epsilon)$ and $\dot{f}\left(0^{-}\right)=\lim _{\epsilon \rightarrow 0^{-}} \dot{f}(\epsilon)$.

Similar to the exponential function frequently used in the solutions of integer-order systems, a function frequently used in the solutions of fractional-order systems is the MittagLeffler function defined as

$$
E_{\alpha, \beta}(z)=\sum_{k=0}^{\infty} \frac{z^{k}}{\Gamma(k \alpha+\beta)},
$$

where $\alpha$ and $\beta$ are arbitrary complex numbers. When $\beta=1$ and $\alpha>0$, (5) becomes $E_{\alpha}(z)=\sum_{k=0}^{\infty} \frac{z^{k}}{\Gamma(k \alpha+1)}$.

\section{Stability ANALysis of CONSENSUS Algorithms FOR FRACTIONAL-ORDER SYSTEMS}

In this section, we derive the conditions on the communication graph and the fractional order such that consensus for fractional-order systems is achieved over a directed communication graph. To study this problem, we introduce a general model of consensus for fractional-order systems by summarizing the following three different cases:

(i) Fractional-order agent dynamics with an integer-order consensus algorithm

Assume that the agent dynamics are

$$
x_{i}^{(\alpha)}(t)=u_{i}(t)
$$

where $x_{i}^{(\alpha)}(t)$ is the $\alpha$ th order derivative of $x_{i}(t)$ with $\alpha \in \mathbb{R}^{+}$and $u_{i}(t)$ is the control input. ${ }^{1}$ An integer-order consensus algorithm is given by (2).

(ii) Integer-order agent dynamics with a fractional-order consensus algorithm

Assume that the agent dynamics are given by (1). A fractional-order consensus algorithm is proposed as

$$
u_{i}(t)=\sum_{j \in N_{i}} a_{i j}\left[x_{j}^{(\beta)}(t)-x_{i}^{(\beta)}(t)\right], \quad \beta \in \mathbb{R}
$$

(iii) Fractional-order agent dynamics with a fractional-order consensus algorithm

Assume that the agent dynamics are given by (6). A fractional-order consensus algorithm is given by (7), where $\alpha \in \mathbb{R}^{+}$and $\beta \in \mathbb{R}$.

When applying the Caputo derivative to (6) and (7), it follows that Cases $(i i)$ and $(i i i)$ can be written as Case (i) by applying the operator ${ }_{a}^{C} D_{t}^{-\beta}$ on both sides of the corresponding system. Therefore, the model in Case $(i)$ can be considered a general model. For a system with $n$ agents, using (2), (6) can be written in matrix form as

$$
{ }_{a}^{C} D_{t}^{\alpha} X(t)=-L X(t),
$$

where $X(t)$ and $L$ are defined in (3). Although the dynamics for a given system are fixed, $\alpha$ in the general model (8) can be changed by choosing consensus algorithms with different fractional orders.

Note that $L$ can be written in Jordan canonical form as

$$
L=P \underbrace{\left[\begin{array}{cccc}
\Lambda_{1} & 0 & \cdots & 0 \\
0 & \Lambda_{2} & \cdots & 0 \\
\cdots & \cdots & & \\
0 & 0 & \cdots & \Lambda_{k}
\end{array}\right]}_{\Lambda} P^{-1}
$$

where $\Lambda_{m}, m=1,2, \ldots, k$, are standard Jordan blocks. Without loss of generality, let the initial time $a=0$. By defining $Y(t) \triangleq P^{-1} X(t)$, (8) can be written as

$$
{ }_{0}^{C} D_{t}^{\alpha} Y(t)=-\Lambda Y(t) \text {. }
$$

\footnotetext{
${ }^{1}$ For a given system, $\alpha$ is fixed.
} 
Suppose that the diagonal entry of $\Lambda_{i}$ is $\lambda_{i}$ (i.e., an eigenvalue of $L$ ). Noting that the standard Jordan block

$$
\Lambda_{i}=\left[\begin{array}{cccc}
\lambda_{i} & 1 & \cdots & 0 \\
0 & \lambda_{i} & \cdots & 0 \\
\cdots & \cdots & & \\
0 & 0 & \cdots & \lambda_{i}
\end{array}\right]
$$

it follows that (9) can be decoupled into $n$ one-dimensional equations represented by either

$$
{ }_{0}^{C} D_{t}^{\alpha} y_{i}(t)=-\lambda_{i} y_{i}(t)
$$

for the equation corresponding to $\Lambda_{i}$ which has dimension equal to one or the last equation corresponding to $\Lambda_{i}$ which has dimension larger than one, or

$$
{ }_{0}^{C} D_{t}^{\alpha} y_{i}(t)=-\lambda_{i} y_{i}(t)-y_{i+1}(t)
$$

otherwise, where $y_{i}(t)$ is the $i$ th component of $Y(t)$.

Lemma 3.1: When $\operatorname{Re}\left(\lambda_{i}\right) \geq 0$, where $\operatorname{Re}(\cdot)$ denotes the real part of a complex number, the solution of (10) has the following properties:

1. When $\alpha \in\left(0, \frac{2 \theta_{i}}{\pi}\right)$ and $\operatorname{Re}\left(\lambda_{i}\right)>0, \lim _{t \rightarrow \infty} y_{i}(t) \rightarrow 0$ as $t \rightarrow \infty$, where $\theta_{i}=\pi-\arg \left\{\lambda_{i}\right\}$ with $\arg \left\{\lambda_{i}^{\infty}\right\}$ denoting the phase of $\lambda_{i}{ }^{2}$

2. When $\alpha \in(0,1]$ and $\lambda_{i}=0, y_{i}(t) \equiv y_{i}(0), \forall t$.

3. When $\alpha \in(1,2)$ and $\lambda_{i}=0, y_{i}(t)=y_{i}(0)+\dot{y}_{i}(0) t$.

4. When $\alpha \in(2, \infty)$, the system is not stable.

Proof: (Proof of Property 1) By taking the Laplace transform of (10), it can be computed from the Laplace transform of $\mathcal{L}\left\{{ }_{a}^{C} D_{t}^{\alpha} f(t)\right\}$ in Section II-C that

$$
\begin{gathered}
\mathcal{L}\left\{y_{i}(t)\right\}=\frac{y_{i}\left(0^{-}\right) s^{\alpha-1}}{s^{\alpha}+\lambda_{i}}, \alpha \in(0,1] \\
\mathcal{L}\left\{y_{i}(t)\right\}=\frac{y_{i}\left(0^{-}\right) s^{\alpha-1}+\dot{y}_{i}\left(0^{-}\right) s^{\alpha-2}}{s^{\alpha}+\lambda_{i}}, \alpha \in(1,2) .
\end{gathered}
$$

From (12) and (13), it can be seen that the denominator of $\mathcal{L}\left\{y_{i}(t)\right\}$ is $s^{\alpha}+\lambda_{i}$ when $\alpha \in(0,2)$. To ensure that all poles of $\mathcal{L}\left\{y_{i}(t)\right\}$ are in the open left half plane (LHP), it follows from the discussion in [19] that $\alpha \in\left(0, \frac{2\left(\pi-\arg \left\{\lambda_{i}\right\}\right)}{\pi}\right)$, that is, $\alpha \in\left(0, \frac{2 \theta_{i}}{\pi}\right)$, where $\frac{2 \theta_{i}}{\pi}>1$ because $\operatorname{Re}\left(\lambda_{i}\right)>0$, i.e., $\arg \left\{\lambda_{i}\right\} \in\left(-\frac{\pi}{2}, \frac{\pi}{2}\right)$. In particular, when $\lambda_{i} \in \mathbb{R}^{+}, \alpha \in(0,2)$ because $\arg \left\{\lambda_{i}\right\}=0$.

(Proof of Properties 2 and 3) The proofs of Properties 2 and 3 follow from [20].

(Proof of Property 4) See [21].

Lemma 3.2: Assume that continuous function $y_{i+1}(t)$ satisfies $\lim _{t \rightarrow \infty} y_{i+1}(t)=0$. When $\operatorname{Re}\left(\lambda_{i}\right)>0$ (i.e., $\arg \left\{\lambda_{i}\right\} \in$ $\left.\left(-\frac{\pi}{2}, \frac{\pi}{2}\right)\right)$ and $\alpha \in\left(0, \frac{2 \theta_{i}}{\pi}\right)$, where $\theta_{i}=\pi-\arg \left\{\lambda_{i}\right\}$, the solution of (11) satisfies $\lim _{t \rightarrow \infty} y_{i}(t)=0$.

Proof: When $\alpha \in(0,1]$, by taking the Laplace transform of (11), it can be computed from the Laplace transform of $\mathcal{L}\left\{{ }_{a}^{C} D_{t}^{\alpha} f(t)\right\}$ that

$$
\mathcal{L}\left\{y_{i}(t)\right\}=\frac{s^{\alpha-1} y_{i}\left(0^{-}\right)-\mathcal{L}\left\{y_{i+1}(t)\right\}}{s^{\alpha}+\lambda_{i}} .
$$

\footnotetext{
${ }^{2}$ We follow the convention that $\arg \{x\} \in(-\pi, \pi]$ for $x \in \mathbb{C}$.
}

It follows from the proof of Property 1 in Lemma 3.1 that the poles of (14) are in the open LHP when $\alpha \in(0,1]$. By applying the final value theorem of the Laplace transform,

$$
\begin{aligned}
\lim _{t \rightarrow \infty} y_{i}(t) & =\lim _{s \rightarrow 0} s \mathcal{L}\left\{y_{i}(t)\right\} \\
& =\lim _{s \rightarrow 0} \frac{s^{\alpha} y_{i}\left(0^{-}\right)-s \mathcal{L}\left\{y_{i+1}(t)\right\}}{s^{\alpha}+\lambda_{i}} \\
& =0,
\end{aligned}
$$

where we have used the fact $s \mathcal{L}\left\{y_{i+1}(t)\right\}=0$ to derive the last equality because $\lim _{t \rightarrow \infty} y_{i+1}(t)=0$.

When $\alpha \in\left(1, \frac{2 \theta_{i}}{\pi}\right)$, it follows from the proof of Property 1 in Lemma 3.1 that the poles of (14) are also in the open LHP. By taking the Laplace transform of (11), it can be computed from the Laplace transform of $\mathcal{L}\left\{{ }_{a}^{C} D_{t}^{\alpha} f(t)\right\}$ that

$$
\mathcal{L}\left\{y_{i}(t)\right\}=\frac{s^{\alpha-1} y_{i}\left(0^{-}\right)+s^{\alpha-2} \dot{y}_{i}\left(0^{-}\right)-\mathcal{L}\left\{y_{i+1}(t)\right\}}{s^{\alpha}+\lambda_{i}} .
$$

Following a similar discussion for $\alpha \in(0,1]$ gives $\lim _{t \rightarrow \infty} y_{i}(t)=0$.

Theorem 3.1: Let $\lambda_{i}$ be the $i$ th eigenvalue of $L$ and $\theta=\min _{\lambda_{i} \neq 0, i=1,2, \cdots, n} \theta_{i}$, where $\theta_{i}=\pi-\arg \left\{\lambda_{i}\right\}$. For fractional-order system (8), consensus is achieved if the fixed communication graph has a directed spanning tree and $\alpha \in\left(0, \frac{2 \theta}{\pi}\right)$. When $\alpha \in(0,1]$, the solution of (8) satisfies $x_{i}(t) \rightarrow x_{j}(t) \rightarrow \mathbf{p}^{T} X(0)$ as $t \rightarrow \infty$, where $\mathbf{p}$ is the left eigenvector of $L$ associated with the zero eigenvalue satisfying $\mathbf{p}^{T} \mathbf{1}=1$. When $\alpha \in\left(1, \frac{2 \theta}{\pi}\right)$, the solution of (8) satisfies $x_{i}(t) \rightarrow x_{j}(t) \rightarrow \mathbf{p}^{T} X(0)+\mathbf{p}^{T} \dot{X}(0) t$ and $\dot{x}_{i}(t) \rightarrow \dot{x}_{j}(t) \rightarrow \mathbf{p}^{T} \dot{X}(0)$ as $t \rightarrow \infty$.

Proof: Noting that the communication graph has a directed spanning tree, it follows from Lemma 2.1 that $L$ has a simple zero eigenvalue and all other eigenvalues have positive real parts. Without loss of generality, let $\lambda_{1}=0$ and $\operatorname{Re}\left(\lambda_{i}\right)>$ $0, i \neq 1$. When $\alpha \in(0,1]$, because $\lambda_{1}=0$ is a simple zero eigenvalue, $\lambda_{1}$ satisfies (10). It follows from Property 2 in Lemma 3.1 that $y_{1}(t) \equiv y_{1}(0)$. When $\lambda_{i}, i \neq 1$, satisfies (10), it follows from Property 1 in Lemma 3.1 that $\lim _{t \rightarrow \infty} y_{i}(t)=0, i \neq 1$. When $\lambda_{i}, i \neq 1$, satisfies (11), it follows from Lemma 3.2 that $\lim _{t \rightarrow \infty} y_{i}(t)=0, i \neq 1$, as well because $y_{i+1}(t)$ also satisfies either (10) or (11), which implies $\lim _{t \rightarrow \infty} y_{i+1}(t)=0$. Combining the above arguments gives $\lim _{t \rightarrow \infty}^{t \rightarrow \infty} Y(t)=\left[y_{1}(0), 0, \cdots, 0\right]^{T}, i \neq 1$, which implies $\lim _{t \rightarrow \infty} X(t)=\lim _{t \rightarrow \infty} P Y(t)=P S Y(0)=P S P^{-1} X(0)$, ${ }_{t \rightarrow \infty}$ where $S=\left[s_{i j}^{t \rightarrow \infty}\right] \in \mathbb{R}^{n \times n}$ has only one nonzero entry $s_{11}=1$. Note that the first column of $P$ can be chosen as 1 while the first row of $P^{-1}$ can be chosen as $\mathbf{p}$ by noting that $\mathbf{1}$ and $\mathbf{p}$ are, respectively, a right and left eigenvector of $L$ associated with $\lambda_{1}=0$ and $\mathbf{p}^{T} \mathbf{1}=1$. Therefore, $\lim _{t \rightarrow \infty} X(t)=P S P^{-1} X(0)=\mathbf{1} \mathbf{p}^{T} X(0)$, that is, $\lim _{t \rightarrow \infty} x_{i}(t) \stackrel{t \rightarrow \infty}{=} \mathbf{p}^{T} X(0)$.

When $\alpha \in\left(1, \frac{2 \theta}{\pi}\right)$, similar to the previous discussion for $\alpha \in(0,1], \lambda_{1}$ satisfies (10). It follows from Property 3 in Lemma 3.1 that $y_{1}(t)=y_{1}(0)+\dot{y}_{1}(0) t$. Because $\operatorname{Re}\left(\lambda_{i}\right)>$ 
$0, i \neq 1$, similar to the previous discussion for $\alpha \in(0,1]$, it follows from Property 1 in Lemma 3.1 and Lemma 3.2 that $\lim _{t \rightarrow \infty} y_{i}(t)=0, i \neq 1$. Therefore, it follows that $\lim _{t \rightarrow \infty} Y(t)=$ $\left[y_{1}(0)+\dot{y}_{1}(0) t, 0, \cdots, 0\right]^{T}$, which implies that $\lim _{t \rightarrow \infty} \dot{Y}(t)=$ $\left[\dot{y}_{1}(0), 0, \cdots, 0\right]^{T}$. Similar to the proof for $\alpha \in(0,1]$, it follows directly that $\lim _{t \rightarrow \infty} x_{i}(t)=\mathbf{p}^{T} X(0)+\mathbf{p}^{T} \dot{X}(0) t$ and $\lim _{t \rightarrow \infty} \dot{x}_{i}(t)=\mathbf{p}^{T} \dot{X}(0)$.

Corollary 3.2: Assume that the fixed communication graph is undirected. For fractional-order system (8), consensus is achieved if the communication graph is connected and $\alpha \in(0,2)$. The consensus equilibria when $\alpha \in(0,1]$ and $\alpha \in\left(1, \frac{2 \theta}{\pi}\right)$ are the same as those in Theorem 3.1.

Proof: When the undirected communication graph is connected, it follows that there is a simple zero eigenvalue and other eigenvalues are positive, which implies that $\theta=\pi$. The statements then follow from the proof in Theorem 3.1.

Note that $\theta$ is closely related to the eigenvalues of $L$, which are also related to the number of agents. In the following, we characterize the relationship between $\alpha$ and the number of agents to ensure consensus.

Lemma 3.3: [22] Let $\lambda_{i}$ be the $i$ th eigenvalue of $L$, then $\arg \left\{\lambda_{i}\right\} \in\left[-\frac{\pi}{2}+\frac{\pi}{n}, \frac{\pi}{2}-\frac{\pi}{n}\right]$ for all $\lambda_{i} \neq 0$.

Theorem 3.3: Assume that there are $n$ agents with $n \geq 2$. For fractional-order system (8), consensus is achieved if the fixed communication graph has a directed spanning tree and $\alpha \in\left(0,1+\frac{2}{n}\right)$.

Proof: It follows from Lemma 3.3 that $\arg \left\{\lambda_{i}\right\} \in\left[-\frac{\pi}{2}+\right.$ $\left.\frac{\pi}{n}, \frac{\pi}{2}-\frac{\pi}{n}\right]$, which implies $\frac{2 \theta}{\pi} \geq 1+\frac{2}{n}$. Therefore, the statement holds apparently from Theorem 3.1.

\section{COMPARISON BETWEEN CONSENSUS FOR FRACTIONAL-ORDER AND INTEGER-ORDER SYSTEMS}

In this section, we discuss the difference between consensus for fractional-order systems and integer-order systems. To study this problem, we first derive the solutions of (10) and (11).

For $\alpha \in(0,1]$, the Laplace transform of (10) is (12). Taking the inverse Laplace transform of (12) gives

$$
y_{i}(t)=y_{i}\left(0^{-}\right) E_{\alpha}\left(-\lambda_{i} t^{\alpha}\right) .
$$

Similarly, for $\alpha \in(1,2)$, the solution of (10) is given by

$$
y_{i}(t)=y_{i}\left(0^{-}\right) E_{\alpha}\left(-\lambda_{i} t^{\alpha}\right)+\dot{y}_{i}\left(0^{-}\right) t E_{\alpha, 2}\left(-\lambda_{i} t^{\alpha}\right) .
$$

For $\alpha \in(0,1]$, the Laplace transform of (11) is (14). Taking the inverse Laplace transform of (14) gives

$$
y_{i}(t)=y_{i}\left(0^{-}\right) E_{\alpha}\left(-\lambda_{i} t^{\alpha}\right)-y_{i+1}(t) *\left[t^{\alpha-1} E_{\alpha, \alpha}\left(-\lambda_{i} t^{\alpha}\right)\right],
$$

where $*$ denotes the convolution operation. Similarly, for $\alpha \in$ $(1,2)$, the solution of $(11)$ is given by

$$
\begin{aligned}
y_{i}(t)= & y_{i}\left(0^{-}\right) E_{\alpha}\left(-\lambda_{i} t^{\alpha}\right)+\dot{y}_{i}\left(0^{-}\right) t E_{\alpha, 2}\left(-\lambda_{i} t^{\alpha}\right) \\
& -y_{i+1}(t) *\left[t^{\alpha-1} E_{\alpha, \alpha}\left(-\lambda_{i} t^{\alpha}\right)\right] .
\end{aligned}
$$

It thus follows that the decaying speeds of MittagLeffler functions determine the speed at which $y_{i}(t)$, where $\operatorname{Re}\left\{\lambda_{i}\right\}<0$, approaches zero. As a result, it follows that the

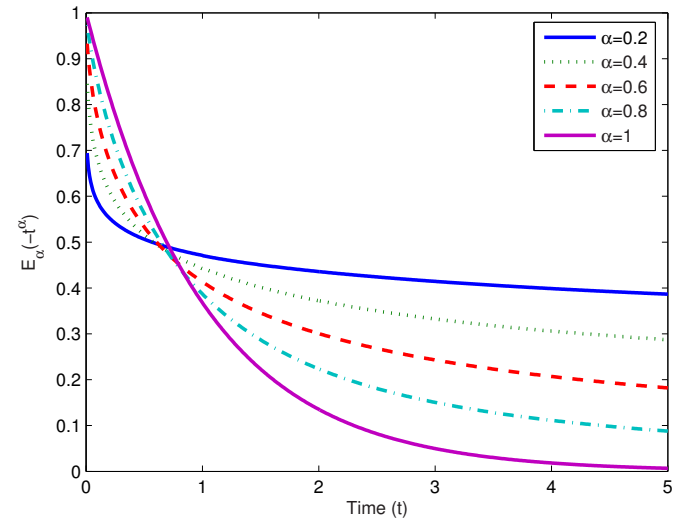

Fig. 1. Mittag-Leffler functions with different orders

convergence speed of (8) is also determined by the decaying speeds of Mittag-Leffler functions due to the fact shown in the proof of Theorem 3.1 that consensus is achieved if $y_{i}(t)=0$ for all $\lambda_{i} \neq 0$. As a special case, when $\alpha=1$, (8) becomes (3) and the Mittag-Leffler functions in the solutions of (10) and (11) become exponential functions. To study the difference between consensus for fractional-order dynamics and integer-order dynamics, the decaying speeds of the Mittag-Leffler function $E_{\alpha}\left(-\lambda t^{\alpha}\right)$ and exponential function $e^{-\lambda t}$ will be discussed in the following as an example.

Theorem 4.1: There exists a positive scalar $T$ such that $E_{\alpha}\left(-\lambda t^{\alpha}\right)$ decreases faster than $e^{-\lambda t}$ for $t \in(0, T)$, where $\lambda \in \mathbb{R}^{+}$and $\alpha \in \mathbb{R}^{+}$.

Proof: Note that both $e^{-\lambda t}$ and $E_{\alpha}\left(-\lambda t^{\alpha}\right)$ equal to 1 when $t=0$. Taking derivatives of both functions gives

$$
\begin{gathered}
\left.\frac{d}{d t}\left[e^{-\lambda t}\right]\right|_{t=0}=-\left.\lambda e^{-\lambda t}\right|_{t=0}=-\lambda, \\
\left.\frac{d}{d t}\left[E_{\alpha}\left(-\lambda t^{\alpha}\right)\right]\right|_{t=0}=-\infty, \alpha>0 .
\end{gathered}
$$

Because $\frac{d}{d t}\left[e^{-\lambda t}\right]$ and $\frac{d}{d t}\left[E_{\alpha}\left(-\lambda t^{\alpha}\right)\right]$ are continuous with respect to $t$, there exists a positive scalar $T$ such that $E_{\alpha}\left(-\lambda t^{\alpha}\right)$ decreases faster than $e^{-\lambda t}$ for $t \in(0, T)$ by using the comparison principle.

To illustrate, Figs. 1 and 2 show Mittag-Leffler functions and their derivatives with different orders for $\lambda=1 .^{3}$ From Fig. 2, we can observe that the fractional-order system always converge faster than the integer-order system when $t<T$ for some positive $T$. This property is desirable in realworld applications. For example, the coordination of a group of vehicles (aircraft/robots) requires a higher convergence speed when the deviations among vehicles are relatively large. However, when the deviations become small, it would be more realistic to decrease the convergence speed because of the mass inertia. Therefore, the proposed fractional-order consensus algorithm is practically useful.

Remark 4.2: In order to achieve higher consensus speed, a varying-order fractional-order consensus strategy can be

\footnotetext{
${ }^{3}$ When $\alpha=1$, the corresponding Mittag-Leffler function becomes the exponential function.
} 


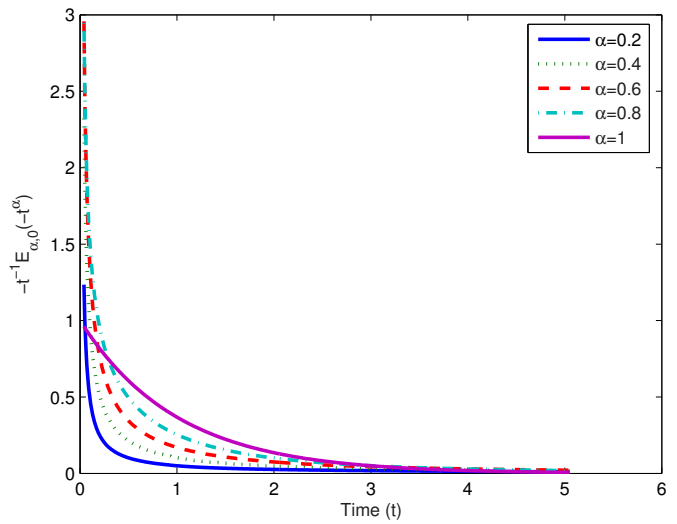

Fig. 2. Derivatives of Mittag-Leffler functions with different orders

adopted. The strategy can be described as follows: Let $\alpha_{1}<$ $\cdots<\alpha_{m}<1$ and choose $\alpha$ in (8) as

$$
\alpha= \begin{cases}\alpha_{1}, & t<t_{1} \\ \alpha_{i}, & t_{i-1} \leq t<t_{i}, i=2, \cdots, m \\ 1, & t \geq t_{m}\end{cases}
$$

Here $t_{1}$ is chosen such that the convergence speed with order $\alpha_{1}$ is the highest when $t<t_{1}$. Similarly, $t_{i}, i=2, \cdots, m$, is chosen such that the convergence speed with order $\alpha_{i}$ is highest for $t \in\left[t_{i-1}, t_{i}\right)$, and $\alpha=1$ if $t \geq t_{m}$. Given the same $L$, the convergence speed of this varying-order fractional-order consensus strategy is higher than that of the single-integrator consensus strategy because the convergence speed of the proposed strategy is higher than that of the single-integrator consensus strategy for $t<t_{m}$ and equal to that of the single-integrator consensus strategy for $t \geq t_{m}$.

\section{Simulation ILLUSTRATIONS AND DisCUSSIONS}

In this section, several simulation results are presented to illustrate the proposed fractional-order consensus algorithm introduced in Section III. We consider a group of four agents. The corresponding Laplacian matrix is chosen as

$$
L=\left[\begin{array}{cccc}
1 & -1 & 0 & 0 \\
0 & 1 & -1 & 0 \\
-1 & 0 & 1 & 0 \\
-1 & 0 & 0 & 1
\end{array}\right]
$$

Note that the communication graph associated with $L$ has a directed spanning tree. It can be computed that $\mathbf{p}=$ $\left[\frac{1}{3}, \frac{1}{3}, \frac{1}{3}, 0\right]^{T}$ and the eigenvalues of $L$ are 0,1 and $1.5 \pm$ $0.866 j$, where $j$ is the imaginary unit.

For $\alpha \in(0,1]$, let the initial states be $X(0)=$ $[6,3,1,-3]^{T}$. When the fractional order is $\alpha=0.8$, the states are shown in Fig. 3(a). It can be seen that consensus is achieved with the final consensus equilibrium for $x_{i}(t)$ being $\frac{10}{3}$, which is equal to $\mathbf{p}^{T} X(0)$. When $\alpha=1$ (i.e., the system takes in the form of single-integrator dynamics), the states are shown in Fig. 3(b). From these two figures, it can be observed that the convergence speed of the fractional-order case is higher than that of the single-integrator case when $t$ is close to the origin.
For $\alpha \in\left(1, \frac{2 \theta}{\pi}\right)$, let $X(0)=[6,3,1,-3]^{T}$ and $\dot{X}(0)=$ $[1,2,3,4]^{T}$. It follows from the definition of $\theta$ in Theorem 3.1 that $\theta=\frac{5 \pi}{6}$, which implies $\alpha \in\left(0, \frac{5}{3}\right)$. Figures 3(c) and 3(d) show the states for $\alpha=1.5$ and $\alpha=1.9$, respectively. From Fig. 3(c), it can be observed that consensus is achieved with the final equilibrium for $\dot{x}_{i}(t)$ being 2 , which is equal to $\mathbf{p}^{T} \dot{X}(0)$. From Fig. 3(d), it can be observed that consensus cannot be achieved.

The states using the varying-order fractional-order consensus strategy are shown in Fig. 4, where we arbitrarily choose $t_{i}=0.1 i$ and $\alpha_{i}=0.2 i$ for $i=1,2,3,4$. From Fig. 4, we can see that the convergence speed using the varying-order fractional-order consensus strategy is higher than that of the integer-order consensus strategy as shown in Fig. 3(b). Of course, when we choose different parameters $\left(\alpha_{i}, t_{i}\right)$ carefully as described in Remark 4.2 , the convergence speed can be further improved.

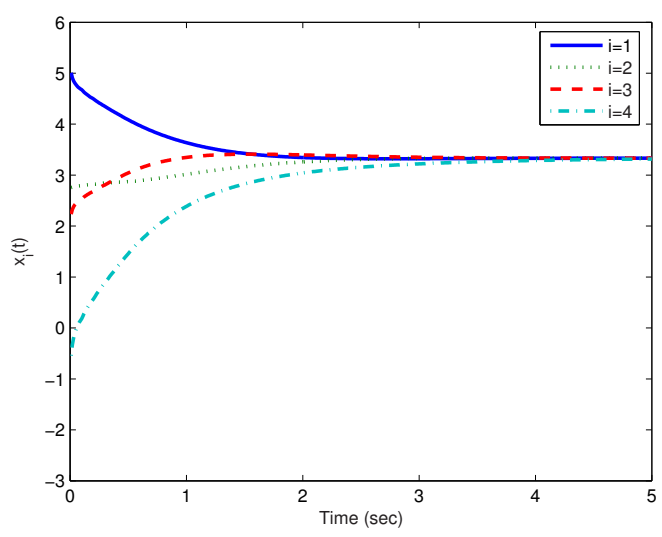

Fig. 4. Simulation result of (8) with varying orders.

\section{CONCLUSion AND Future WORK}

In this paper, we studied distributed coordination algorithms for multiple fractional-order systems when the fixed communication graph is directed. We presented a general fractional-order consensus model and then investigated its stability properties. Sufficient conditions on the communication graph and the fractional order were given to ensure consensus. In addition, we characterized the relationship between the fractional order and the number of agents to ensure consensus. Finally, a varying-order fractional-order consensus strategy was proposed to improve the overall convergence speed. Future work will explore fractionalorder consensus algorithms with time-varying or switching communication graphs.

\section{REFERENCES}

[1] R. L. Winkler, "The consensus of subjective probability distributions," Manage Science, vol. 15, no. 2, pp. B61-B75, October 1968.

[2] M. H. DeGroot, "Reaching a consensus," Journal of American Statistical Association, vol. 69, no. 345, pp. 118-121, 1974.

[3] T. Vicsek, A. Czirok, E. B. Jacob, I. Cohen, and O. Schochet, "Novel type of phase transitions in a system of self-driven particles," Physical Review Letters, vol. 75, no. 6, pp. 1226-1229, 1995. 


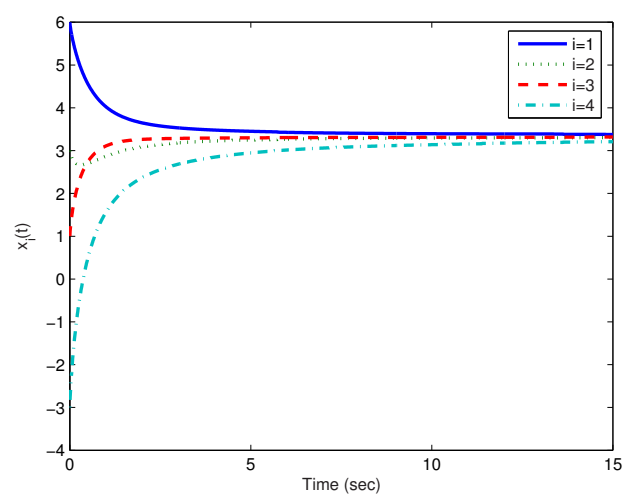

(a) $\alpha=0.8$.

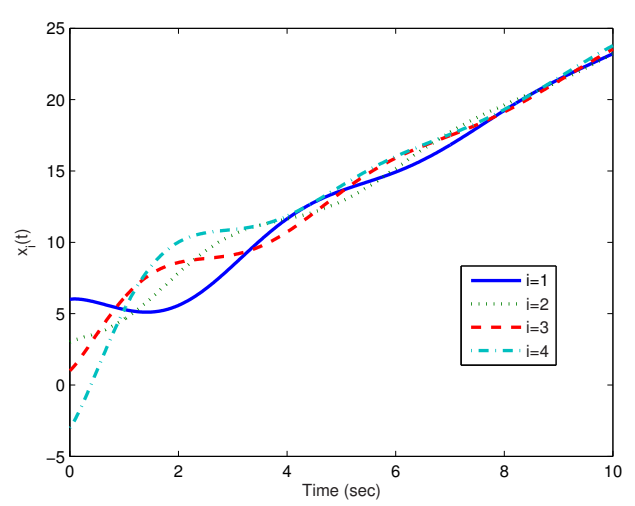

(c) $\alpha=1.5$.

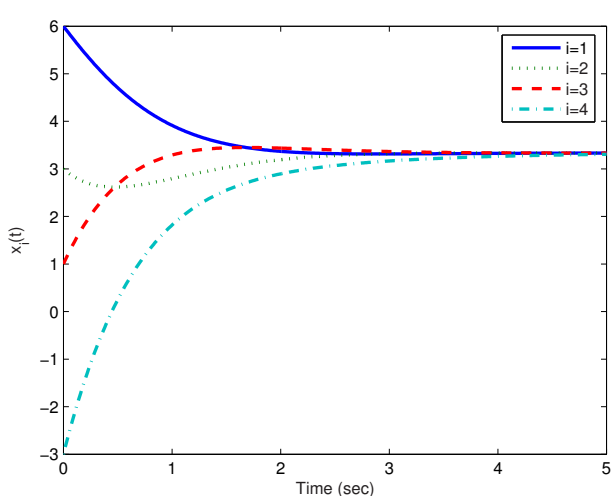

(b) $\alpha=1$.

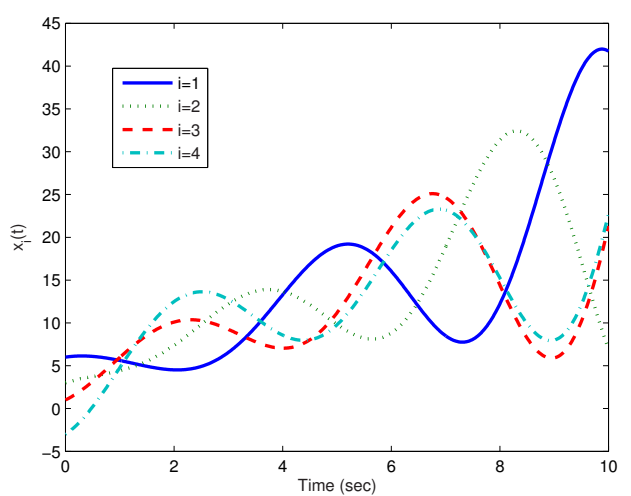

(d) $\alpha=1.9$.

Fig. 3. Simulation results of (8) with different orders.

[4] W. Ren, R. W. Beard, and E. M. Atkins, "Information consensus in multivehicle cooperative control: Collective group behavior through local interaction," IEEE Control Systems Magazine, vol. 27, no. 2, pp. 71-82, April 2007.

[5] R. Olfati-Saber, "Flocking for multi-agent dynamic systems: Algorithms and theory," IEEE Transactions on Automatic Control, vol. 51, no. 3, pp. 401-420, March 2006.

[6] G. Xie and L. Wang, "Consensus control for a class of networks of dynamic agents," vol. 17, no. 10-11, pp. 941-959, July 2007.

[7] W. Ren and E. M. Atkins, "Distributed multi-vehicle coordinated control via local information exchange," International Journal of Robust and Nonlinear Control, vol. 17, no. 10-11, pp. 1002-1033, July 2007.

[8] H. G. Tanner, A. Jadbabaie, and G. J. Pappas, "Flocking in fixed and switching networks," IEEE Transactions on Automatic Control, vol. 52, no. 5, pp. 863-868, May 2007.

[9] D. Lee and M. W. Spong, "Stable flocking of multiple inertial agents on balanced graphs," IEEE Transactions on Automatic Control, vol. 52, no. 8, pp. 1469-1475, August 2007.

[10] R. L. Bagley and P. J. Torvik, "Fractional calculus - a different approach to the analysis of viscoelastically damped structures," AIAA J., vol. 21, no. 5, pp. 741-748, 1983.

[11] _ _ "A theoretical basis for the application of fractional calculus to viscoelasticity," Journal of theology, vol. 27, no. 3, pp. 201-210, 1983.

[12] — , "On the fractional calculus model of viscoelastic behavior," Journal of theology, vol. 30, no. 1, pp. 133-155, 1986.

[13] Y. Kozlovsky, I. Cohen, I. Golding, and E. Ben-Jacob, "Lubricating bacteria model for branching growth of bacterial colonies," 1999. [Online]. Available: citeseer.ist.psu.edu/kozlovsky99lubricating.html

[14] I. Cohen, I. Golding, I. G. Ron, and E. Ben-Jacob, "Bio-uiddynamics of lubricating bacteria," Math. Meth. Appl. Sci, vol. 24, pp. 1429-1468, 2001.

[15] W. Ren and R. W. Beard, "Consensus seeking in multiagent systems under dynamically changing interaction topologies," IEEE Transactions on Automatic Control, vol. 50, no. 5, pp. 655-661, May 2005.

[16] A. Jadbabaie, J. Lin, and A. S. Morse, "Coordination of groups of mobile autonomous agents using nearest neighbor rules," IEEE Transactions on Automatic Control, vol. 48, no. 6, pp. 988-1001, June 2003.

[17] R. Olfati-Saber and R. M. Murray, "Consensus problems in networks of agents with switching topology and time-delays," IEEE Transactions on Automatic Control, vol. 49, no. 9, pp. 1520-1533, September 2004.

[18] W. Ren, K. L. Moore, and Y. Chen, "High-order and model reference consensus algorithms in cooperative control of multi-vehicle systems," ASME Journal of Dynamic Systems, Measurement, and Control, vol. 129, no. 5, pp. 678-688, 2007.

[19] Y. Chen, H.-S. Ahn, and I. Podlubny, "Robust stability check of fractional order linear time invariant systems with interval uncertainties," (Elsevier) Signal Processing, vol. 86, pp. 2611-2618, March 2006.

[20] I. Podlubny, Fractional Differential Equations. Academic press, 1999.

[21] R. Gorenflo and F. Mainardi, "Fractional oscillations and mittagleffler functions," 1996. [Online]. Available: citeseer.ist.psu.edu/ gorenflo96fractional.html

[22] N. Dmitriev and E. Dynkin, "On the characteristic numbers of a stochastic matrix," C.R. (Doklady) Acad. Sci. URSS, vol. 49, no. 3, pp. 159-162, 1946. 